Para enlazar con este artículo / To link to this article:

http://dx.doi.org/10.6035/MonTI.2021.13.07

Para citar este artículo / To cite this article:

MejíAS-CLIMENT, Laura. (2021) "Los estudios de corpus y la localización: Una propuesta de análisis para material interactivo." En: CAlZADA, María \& Sara LAVIOSA (eds.) 2021. Reflexión crítica en los estudios de traducción basados en corpus / CTS spring-cleaning: A critical reflection. MonTI 13, pp. 220-250.

\title{
LOS ESTUDIOS DE CORPUS Y LA LOCALIZACIÓN: UNA PROPUESTA DE ANÁLISIS PARA MATERIAL INTERACTIVO
}

\author{
LAURA MejÍAS-CLIMENT \\ lmejias@uji.es \\ Universitat Jaume I
}

\section{Resumen}

Este artículo pretende dar cuenta de las sincronías empleadas en el doblaje al español peninsular de un corpus multimodal compuesto por tres videojuegos del género interactivo de la acción-aventura. La metodología, de enfoque descriptivo, triangula datos cualitativos y cuantitativos obtenidos, por una parte, del análisis empírico del corpus multimodal y, por otra, del contacto directo con profesionales de la industria mediante entrevistas semiestructuradas. Asimismo, se revisarán algunos planteamientos previos de los estudios de corpus, estrechamente relacionados con los Estudios Descriptivos en Traducción, y de la práctica profesional de la localización, abordándola desde el enfoque de la Traducción Audiovisual (TAV). Se busca así exponer cómo combinar distintos métodos y perspectivas para analizar la modalidad de TAV del doblaje en un producto multimodal interactivo, aspecto escasamente investigado empíricamente en la esfera académica por el momento, a pesar de la utilidad que ya han demostrado los estudios de corpus en el panorama traductológico.

Palabras clave: Corpus multimodal; Interacción; Localización; Doblaje; Videojuegos.

\begin{abstract}
This article aims to analyze the dubbing synchronies used in a multimodal corpus composed of three video games, dubbed into Castilian Spanish, belonging to the interactive genre of action-adventure. The methodology, adopting a descriptive approach,
\end{abstract}


triangulates qualitative and quantitative data obtained, on the one hand, from the empirical analysis of the multimodal corpus and, on the other hand, from direct contact with professionals in the industry through semi-structured interviews. Additionally, some previous approaches within Corpus-Based Translation Studies_closely linked to Descriptive Translation Studies-will be reviewed, as well as the professional practice of localization, from the perspective of audiovisual translation (AVT). The goal is thus to present how different methods and perspectives can be combined to analyze the AVT mode of dubbing in a multimodal and interactive product, which remains largely unexplored in academia so far, despite the efficacy that corpus studies have demonstrated in translation studies.

Keywords: Multimodal corpus; Interaction; Localization; Dubbing; Video games.

\section{El poliédrico marco de trabajo de los Estudios de Traducción}

Uno de los atractivos del ámbito de la traducción es la versatilidad de enfoques, métodos y áreas que este abarca, tanto profesionales como investigadores. Esto nos permite diseñar modelos de investigación como el que se presenta en estas páginas, cuyo principal objetivo es determinar los tipos de ajuste reflejados en las situaciones de juego de un corpus compuesto por tres videojuegos del género interactivo de la acción-aventura: Batman: Arkham Knight (Rocksteady Studios, 2015), Assassin's Creed Sydicate (Ubisoft, 2015) y Rise of the Tomb Raider (Crystal Dynamics, 2016). Para ello, describiremos a continuación el planteamiento teórico y los estudios previos en los que se fundamenta nuestro diseño, así como la metodología empleada y los resultados obtenidos, de carácter tanto cuantitativo como cualitativo.

En las últimas décadas hemos presenciado el arraigo de los Estudios de Traducción basados en Corpus desde el artículo de referencia de Baker en 1993, que ponía de manifiesto la utilidad de la lingüística de corpus para la traducción (Zanettin 2014). La metodología basada en corpus se ha asociado estrechamente con los Estudios Descriptivos en Traducción, que gozan de más recorrido en el mundo académico desde las primeras publicaciones de los años 70 (Hermans 2020). Hasta nuestros días, han sido numerosísimas las propuestas metodológicas tanto en el uso de corpus como en los planteamientos descriptivos. Nos remitimos a Bernardini \& Kenny (2020) 
y Hermans (2020), respectivamente, para una sucinta revisión de las principales publicaciones asociadas a ambos campos.

Por otra parte, desde el prisma profesional, el desarrollo de las tecnologías ha traído consigo el establecimiento de la localización como una potente industria para la adaptación, tanto cultural como lingüística, legal y, en conjunto, funcional y cultural de todo producto multimedia (Jiménez-Crespo 2020). Sus orígenes se remontan a finales de la década de los setenta. Desde su consolidación entre los 80 y los 90 hasta el presente, la localización se ha desarrollado a un ritmo vertiginoso y se asocia con la adaptación de tres grandes grupos de productos: contenidos web, software y videojuegos (Mata Pastor 2005), además de los dispositivos móviles o small devices que añade Jiménez-Crespo (2013b).

Dichos productos, entre otros muchos, pueden considerarse ejemplos de textos multimodales (Kress y Van Leeuwen 2001), pues combinan múltiples modos semióticos integrados para crear su sentido conjunto. Aunque se trata de un concepto sobre el cual aún quedan cuestiones clave en las que alcanzar un consenso (Pérez-González 2020), la multimodalidad, desde su incursión en el ámbito académico en la década de los 90 (Jewitt, Bezemer y O'Halloran 2016), ha permitido el análisis holístico de la comunicación, teniendo en cuenta no únicamente el código lingüístico, sino también todo modo semiótico que lo acompaña y completa, ya sea visual, acústico, kinésico, etc. (Caple 2018).

De especial utilidad en el actual panorama multimedia ha demostrado ser la convergencia entre multimodalidad y estudios de corpus, mediante el análisis de corpus multimodales o MMC (Soffritti 2018), para dar cuenta de la configuración semiótica completa de los productos multimedia. En los MMC nos centraremos en estas páginas, con la elección de los videojuegos como producto multimodal y, además, interactivo, sobre el que aún quedan numerosísimos rincones que explorar (O'Hagan y Mangiron 2013).

Por último, un entorno más al que debemos referirnos, y en estrecha relación con la localización, con el uso de corpus y con la multimodalidad, es la Traducción Audiovisual (TAV), algo más antigua que la práctica localizadora, ya que surgió de la mano de la industria cinematográfica (Díaz Cintas y Anderman 2009) y, desde entonces, su flexibilidad ha sido evidente (Orero 2004) a la hora de acomodarse al amplio y cambiante mercado de productos 
audiovisuales, no solo desde la práctica profesional, sino también con una gran variedad de planteamientos investigadores (Chaume 2018a).

Aprovechando, como se decía, la versatilidad de la traducción, todas estas perspectivas confeccionan el marco de interés del presente artículo, con el que pretendemos ofrecer un planteamiento original, empleando perspectivas mixtas, en la combinación de los Estudios Descriptivos y basados en Corpus, con la práctica de la localización y el ámbito de la TAV.

Dado el amplio alcance que tanto la localización como la TAV ofrecen, nos centraremos en una modalidad de TAV, el doblaje, escasamente explorada hasta ahora en la práctica localizadora profesional (O'Hagan y Mangiron 2013). Del mismo modo, también son "escasos [los] estudios sobre el uso de corpus en localización” (Jiménez-Crespo 2009: en línea), motivo por el cual hemos decidido basar la investigación en un corpus multimodal compuesto por los tres citados videojuegos.

Para delimitar el estudio de dicho corpus, nos centraremos en analizar las sincronías, una de las características principales del doblaje. Así, partiremos del polémico nexo entre TAV y localización para exponer la metodología particular que se ha diseñado para trabajar empíricamente con un corpus multimodal de videojuegos, cuya originalidad con respecto a estudios previos radica en la contemplación de una dimensión interactiva y en el uso de las situaciones de juego como unidad de análisis.

Además de la complejidad que ya presentan los estudios basados en corpus multimodales, en los que deben tenerse en cuenta diversos modos de transmisión del sentido (Soffritti 2018), en este caso sumamos un canal táctil no explorado en estudios empíricos en traducción hasta ahora, para lo cual se propone adaptar el planteamiento analítico a la medida de los intereses de la investigación y de la idiosincrasia del producto estudiado.

La complejidad aquí va más allá al contemplar una dimensión interactiva. Reconocemos que el diseño de esta investigación, tal y como señalan Taylor y Marchi (2018: 11), podría influir en la dirección que tome el análisis y en los resultados obtenidos. Por ello, hemos querido recurrir a la triangulación de datos para completar la información empírica con información procedente de los testimonios de profesionales del sector. No obstante, se trata de un diseño preliminar en el que se identificarán algunos puntos fuertes y débiles 
a lo largo de las siguientes páginas y que puede tomarse como estudio de partida para refinar el procedimiento en el futuro.

Dado que este análisis contempla diversos campos y enfoques dentro de los Estudios de Traducción, los siguientes apartados reflejan el fundamento poliédrico de nuestro trabajo: la TAV y la localización como ámbitos profesionales analizados, los estudios de corpus como enfoque metodológico $y$, por último, los textos interactivos como producto multimodal que ofrece aún numerosas aristas de análisis.

\section{Traducción Audiovisual y localización: encuentros y desencuentros}

Ya desde comienzos de siglo (Orero 2004) y más aún en nuestros días (Chaume 2018b), la TAV parece funcionar como un concepto de gran amplitud bajo el que situar muy diversas modalidades de traducción, entendidas según los métodos técnicos empleados para trasvasar el código lingüístico de un texto audiovisual de partida a uno meta (Chaume 2004a: 31) o el modo del discurso del texto original y del meta (Hurtado Albir 2011: 69-70).

Tradicionalmente, la TAV engloba a aquellas modalidades que añaden una banda sonora al producto original (revoicing) y aquellas basadas en insertar texto en la pantalla en la que se proyecta el texto audiovisual de partida (captioning) (Chaume 2018b). En este caso, nos centraremos en la modalidad de revoicing de mayor tradición histórica en nuestro país: el doblaje.

Orero (2004: VIII) ya llamaba la atención sobre la flexibilidad del concepto de TAV para abarcar tantas diversas formas de transferencia multisemiótica como las nuevas tecnologías trajeran consigo:

Audiovisual Translation will encompass all translations - or multisemiotic transfer - for production or postproduction in any media or format, and also the new areas of media accessibility [...]. Technological developments which have changed paper oriented society towards media oriented society have also made Audiovisual Translation the most dynamic field of Translation Studies.

En el presente, además de transferencias inter e intralingüísticas, contamos también con la intersemiótica (Jakobson 2000), con modalidades de TAV como la audiodescripción o las audioguías para museos; también puede ubicarse bajo el paraguas de la TAV la transadaptación (Gambier 2003, 
Neves 2005, Pruys 2009), la transcreación (O'Hagan y Mangiron 2013, Bernal Merino 2015), las narrativas transmedia (Bernal Merino 2015, Pujol Tubau 2015) y las adaptaciones o remakes, así como la localización. Es aquí donde nos interesa hacer referencia al actual debate sobre TAV y localización como prácticas estrechamente relacionadas entre las cuales los límites no están claros, si es que alguna vez lo han estado (O'Hagan y Mangiron 2013), y menos aún lo están a medida que avanzan los medios tecnológicos con nuevos productos y géneros.

Por una parte, un sector de los profesionales dedicados a la localización defiende su clara diferenciación de la TAV al concebir la traducción desde una posición reduccionista y meramente lingüística (Cadieux y Esselink 2004, Maxwell-Chandler y Deming 2012). También otros profesionales y algunos investigadores defienden la visible separación de los ámbitos de la localización y la TAV, por tratarse la primera de un proceso industrial particular que abarca muchas más modificaciones y etapas que la traducción (Jiménez-Crespo 2013b, Pym 2014, Muñoz Sánchez 2017, Méndez González y Calvo-Ferrer 2017).

Por otro lado, autores como Bernal Merino (2006) y O'Hagan y Mangiron (2013) adoptan el uso del término localización, dado su extendido empleo en la industria, aunque reconocen que no contempla nada que no abarque ya un sentido amplio de la traducción, por lo que no sería necesaria una estricta separación en dos ámbitos diferenciados.

Ante ambas posturas, la clave parece estar entonces en la perspectiva que se adopte: bien la posición de la industria, que aboga por la diferenciación de la localización, o bien, la académica, donde no parece ser estrictamente necesario crear un nuevo paradigma. En estas páginas, emplearemos el término localización entendiendo tal práctica como la plena adaptación de un producto multimedia (contenidos web, software o videojuegos) a un mercado local meta, en línea con los enfoques de O'Hagan y Mangiron (2013) y Bernal-Merino (2015). Se diferencia así de la práctica profesional de la TAV, entre otros aspectos, en los productos de los que se ocupa y la diversidad de procedimientos que abarca.

En lo que respecta al planteamiento metodológico de este estudio, como se verá en la sección 4, tal concepción de la localización no nos impide situarnos en la misma posición adoptada por Vázquez Rodríguez (2018), 
quien, ante el debate sobre la distinción entre TAV y localización, propone adaptar la metodología descriptivista ya empleada en la TAV para contemplar particularidades que pueda presentar el producto audiovisual y multimedia analizado, sea cual sea - en este caso, el principal rasgo particular es la presencia de la interactividad en un corpus de videojuegos-. Por lo tanto, entendemos la localización y la TAV como prácticas profesionales que se ocupan tradicionalmente de distintos productos, pero cuya convergencia en numerosos aspectos favorece la adopción de metodologías de investigación comunes.

\section{Los Estudios de Traducción basados en Corpus y los productos multimodales}

Tal y como Baños, Bruti y Zanotti (2013) ponen de manifiesto, la lingüística de corpus ha demostrado ampliamente su utilidad en los Estudios de Traducción en general (Baker 1996, Olohan 2004, Laviosa 2012), así como en la TAV en particular (Mangiron 2017), según muestran las contribuciones de Forchini, Baños, Valentini, Valirano, Jiménez Hurtado y Soler Gallego, y Bywood et al. en la citada edición de Baños, Brutti y Zanotti (2013) sobre la aplicación de la lingüística de corpus a la TAV, entre muchísimas otras obras.

A pesar de los grandes avances que los corpus han aportado a la investigación en TAV en términos de generalizaciones descriptivas y mejora de la calidad, aún quedan aspectos en los que trabajar, como la representatividad, el tamaño, la comparabilidad, la transcripción, la anotación y la alineación de estos corpus audiovisuales (Pavesi 2018). Aunque nuestro estudio es aún un punto de partida en el análisis de un pequeño corpus interactivo y adolece de varias limitaciones en las citadas categorías, consideramos que representa una propuesta preliminar original que puede apuntar a nuevas perspectivas en el estudio de los corpus multimodales.

Situar el análisis de videojuegos en el paradigma de la TAV ofrece la ventaja de que, casi desde sus inicios, el objeto de estudio se ha abordado desde un enfoque multimodal (Kaindl 2013: 263), teniendo en cuenta explícitamente tanto elementos verbales como no verbales, aunque, ciertamente, los videojuegos y la interacción que traen con ellos son todavía más recientes, pues su origen puede situarse alrededor de los años 60 (Kent 2001, López 
Redondo 2014) y la metodología de estudio ha de ajustarse a dicha interacción adecuadamente, en el conjunto multimodal.

Mangiron (2017) llama la atención sobre la necesidad de emprender estudios de corpus en el ámbito de la localización. Así, podría recuperarse una notable cantidad de datos sobre tendencias y regularidades en la localización de videojuegos. En este campo se han estudiado ampliamente el proceso (Maxwell-Chandler y Deming 2012, O'Hagan y Mangiron 2013; Bernal Merino 2015), las restricciones (Dietz 2007, Loureiro Pernas 2007, Muñoz Sánchez 2017) y las estrategias de traducción (Fernández Costales 2012), pero no se han desarrollados tantos estudios empíricos basados en corpus como en otros tipos de texto.

Hasta la fecha existen diversos estudios de caso que analizan videojuegos concretos (Crosigniani y Ravetto 2001, Fernández Torné 2007, Mangiron 2010, Ensslin 2012, Müller Galhardi 2014 y Van Oers 2014, entre otros), pero únicamente se han empleado corpus de videojuegos, hasta donde se ha podido comprobar, en la obra de Pujol Tubau (2015) — aunque no exclusivamente, pues su análisis reúne producción transmedia- y en la obra de Vázquez Rodríguez (2018). En ninguno de estos trabajos, sin embargo, se emplean las situaciones de juego como unidades de análisis (véase sección 4.1.), aspecto que suma originalidad a nuestra propuesta.

Tras la introducción de los corpus en los Estudios de Traducción a comienzos de los 90, fue haciéndose patente la necesidad de adoptar enfoques más allá de los puramente descriptivistas y lingüísticos para dar cuenta de la complejidad de la traducción ampliando el marco de estudio mediante su contextualización y la integración de herramientas de análisis de otras áreas (Olohan 2004). La integración de corpus y métodos mixtos de investigación del proceso de traducción ha resultado especialmente fructífera (Malamatidou 2018). Además de diseños de investigación basados en distintos métodos, algunos proyectos recientes han ido expandiéndose hacia fuentes previamente ignoradas, quizá por su complejidad, y producidas bajo condiciones relativamente recientes (Bernardini y Kenny 2020: 113), como es el caso de los trabajos de Jiménez-Crespo (2013a, 2015), centrados en el análisis de corpus compuestos por una modalidad (la localización web) y un género (los sitios de redes sociales) que ni siquiera existían cuando comenzó a sistematizarse el estudio de tendencias en traducción y el uso de corpus. 
En estas nuevas investigaciones, a cuyo paradigma la nuestra pretende sumarse, uno de los aspectos cruciales es la contemplación de la dimensión no verbal y la configuración semiótica multimodal en el diseño del corpus. Combinando la teoría multimodal y los adecuados diseño y consulta del corpus, la investigación puede abrirse a la naturaleza multisemiótica del producto y su impacto en la traducción. Por lo tanto, un enfoque integrador que combine la lingüística de corpus con el análisis multimodal y recursos y fuentes extralingüísticos (entrevistas), triangulando los datos, podrá dar cuenta de la naturaleza híbrida y compleja del producto (Baños, Bruti y Zanotti 2013).

\section{Metodología: triangulación de datos con un corpus interactivo}

A continuación, describiremos el proceso de trabajo con un pequeño corpus paralelo (Laviosa 2002) en el que se han analizado los tipos de ajuste empleados en las versiones de partida, en inglés, y doblada al español peninsular, en los segmentos originales y sus correspondientes traducciones (Toury 1995). Se trata de un corpus multimodal o multimedia (Soffritti 2018), ya que recoge tanto vídeos (imagen y audio) como texto. El corpus abarca 2635 registros con cadenas de texto de entre 2 y 300 palabras (1326 registros en español y 1309 en inglés) que se extienden durante 76 horas de juego. Los datos extraídos del análisis del corpus se triangularán con información procedente de entrevistas semiestructuradas que también presentaremos.

Ante la escasez de estudios académicos que ahondaran en el nexo entre doblaje y localización de videojuegos, el punto de partida de este estudio es la siguiente pregunta de investigación: ¿qué tipos de ajuste pueden asociarse a cada situación de juego presente en videojuegos de acción-aventura? Será necesario ampliar el corpus en el futuro para confirmar los resultados obtenidos aquí, que pueden entenderse como una aproximación inicial a esta pregunta, dada la limitación del corpus a una selección de tres videojuegos de este género. Los métodos que se emplearán para responderla derivarán de las necesidades que la propia pregunta nos plantea (Taylor y Marchi 2018: 3), así como de la creatividad necesaria para enfocar un estudio de corpus tan particular (ibid., 6). 


\subsection{El análisis de los textos interactivos: semiótica y segmentación del corpus}

Al abordar el análisis de un corpus compuesto por videojuegos bajo el amplio paraguas de la TAV es necesario, para comenzar, delimitar el concepto y la configuración semiótica particular del objeto de estudio con el fin de poder diseñar posteriormente el análisis de acuerdo con su idiosincrasia. Ciertamente, se trata del producto audiovisual (y multimodal) actual más complejo, dados los variados modos que coexisten en su entramado semiótico (Maietti 2004).

Como producto multimedia, los videojuegos encajan en la ya conocida concepción de todo texto audiovisual como aquél que se transmite a través de los canales acústico y visual y cuyos diversos códigos se entrelazan para tejer su sentido completo (Chaume 2012). Ahora bien, en el caso de los videojuegos, además de los códigos acústicos y visuales de dichos canales, debe tenerse en cuenta también la dimensión interactiva, que quizás sea la clave en el éxito mundial que han experimentado estos productos en tan solo unas décadas (López Redondo 2014), puesto que convierten al espectador pasivo en figura activa y protagonista de los hechos en pantalla. Así, a la ya conocida configuración semiótica de todo producto audiovisual, en videojuegos debemos sumar un canal táctil (Pujol Tubau 2015) que vehicula códigos hápticos bidireccionales (juego $\leftrightarrow$ jugador) cuando se emplea un mando o algún otro periférico para jugar. Nos remitimos a Mejías-Climent (2019) para una revisión del funcionamiento del canal táctil y otras formas de juego sin mando.

En el caso de los tres videojuegos que componen nuestro corpus, el jugador recibirá información a través del canal táctil mediante códigos hápticos si el mando vibra. Pero, sobre todo, este usuario devolverá información empleando el tacto en movimiento (de nuevo, códigos hápticos en forma de pulsación de botones o palancas en el periférico), que el juego interpretará en función de sus reglas y ante los que reaccionará para completar así la creación del sentido conjunto del videojuego.

En este tipo de productos interactivos, el desarrollo de los hechos depende, por tanto, de la acción continua de quien juega, que va interactuando con el juego y causando la alternancia constante de distintas situaciones de juego (Pujol Tubau 2015, Mejías-Climent 2017), las cuales implican 
diferentes niveles de interacción con el usuario. En concreto, se trata de tareas, diálogos, acción de juego y cinemáticas. Las dos primeras pueden darse durante interacción plena o parcial (el jugador puede recibir tareas o dialogar con otros personajes mientras actúa plenamente o, por el contrario, sus movimientos se limitarán parcialmente, aunque no por completo, para obligarlo a atender a dichas instrucciones o conversaciones); la acción representa la plena interacción del jugador con el producto, mientras que las cinemáticas detienen por completo toda interacción para trasladarnos por unos segundos a un vídeo tradicional que recurre a la configuración cinematográfica.

La estructura audiovisual de un videojuego, por lo tanto, no está cerrada de antemano, como sí sucede en películas o series, de forma que el análisis de un corpus compuesto por productos interactivos habrá de tener en cuenta esta particularidad a la hora de estructurarse. Aprovechando los distintos niveles de interacción y la clara diferenciación de las citadas cuatro situaciones de juego, estas se tomarán como la unidad de análisis que permitirá identificar y situar el fenómeno estudiado dentro del corpus (las sincronías del doblaje) en lugar de recurrir a los códigos de tiempo, como se haría en corpus compuestos por películas o series, de duración y estructura únicas y cerradas.

\subsection{El doblaje como nexo común y delimitador del corpus}

Además de constituir el fenómeno específico que se analiza en este corpus, la sincronía, por su parte, constituye uno de los estándares de calidad que propugna la industria del doblaje en nuestro país (Chaume 2007). Representa la coherencia entre la imagen que se aprecia en pantalla y el elemento sonoro, y se ha clasificado en tres tipos para cine y televisión: sincronía fonética o labial (reproducción de la articulación de los labios de los personajes, siempre que se les vea en primer plano o plano detalle), cinésica (correspondencia de la traducción con los movimientos y expresividad de los personajes) e isocronía (misma duración de los enunciados originales y traducidos) (Chaume 2004b).

En el caso del doblaje de videojuegos, varias son las diferencias reseñables en el proceso y los materiales disponibles en comparación con la práctica 
del doblaje en productos no interactivos (Mejías-Climent 2019). De entre todas ellas, cabe señalar en especial la no disponibilidad de los vídeos que se doblarán, ni para los traductores — que a menudo trabajan con meras restricciones de espacio marcadas en las cadenas de texto descontextualizadas que traducen-, ni para los agentes en sala de doblaje (actores, directores y técnicos de sonido), cuya única referencia, en la mayoría de los casos, son las ondas de audio originales.

La sincronía en videojuegos, por tanto, ha de entenderse como una gradación de restricciones, que pueden aplicarse hasta en cinco niveles (es decir, se contabilizan cinco tipos de ajuste o sincronía) (Pujol Tubau 2015; Mejías-Climent 2018, 2019, 2020). Cada una de ellas implica lo siguiente:

(1) Libre: segmentos traducidos sin restricción (voces en off).

(2) Temporal: los segmentos traducidos pueden ser un 10 o $20 \%$ más cortos o largos que los originales.

(3) Temporal exacto: los segmentos traducidos han de tener exactamente la misma duración que los originales, sin tener en cuenta pausas ni articulaciones internas.

(4) Sonoro: los segmentos traducidos han de tener exactamente la misma duración que los originales y, además, respetar pausas o entonaciones particulares.

(5) Labial: los segmentos traducidos han de ser idénticos a los originales en cuanto a duración y articulación, similar al ajuste labial cinematográfico.

Estos tipos de ajuste guiarán la pregunta principal que se consultará en el corpus, pues se buscará identificar una relación entre situaciones de juego, que implican distintos niveles de interacción, y tipos de ajuste, que pueden ser más o menos restrictivos, según se ha explicado. Los datos que se obtengan serán de carácter cuantitativo, en función de los porcentajes asignados a cada tipo de ajuste identificado para cada situación de juego, lo cual nos permitirá, a su vez, llegar a conclusiones cualitativas sobre los niveles de restricción de las situaciones de juego en relación con las sincronías del doblaje. Esta información se completará con entrevistas a los agentes del proceso (véase apartado 4.4.). 


\subsection{Diseño del estudio: ficha de análisis}

Un estudio empírico para trazar tendencias en el uso de las sincronías del doblaje en un determinado contexto sociocultural requiere, en primer lugar, de un corpus de análisis claramente delimitado sobre el que puedan detectarse los patrones, en este caso, empleados en el doblaje al español peninsular de videojuegos originalmente desarrollados en inglés. Como vemos, se trata de un corpus multimodal paralelo, bilingüe y unidireccional (inglés>español) (Laviosa 2012). Posteriormente, una serie de entrevistas con los principales agentes del proceso de traducción y doblaje nos darán las claves principales sobre el contexto sociocultural en el que se sitúa el corpus.

Siguiendo las fases de la metodología que describe Laviosa (2012: 68), para empezar, se establecieron los criterios para seleccionar el corpus de entre una población de 106 videojuegos comercializados en España, entre 2015 y 2016, con los diálogos sonoros disponibles en español. Para reducir esta población a un catálogo de menor tamaño se añadió el requisito de que la lengua original de desarrollo (y, por tanto, de partida para el doblaje) fuera el inglés. Los 80 videojuegos resultantes, de nuevo, se acotaron aplicando los siguientes filtros: el género interactivo (Wolf 2005; Mejías-Climent 2019) ha de ser la acción-aventura; el modo, visión en 3. ${ }^{a}$ persona — de forma que la perspectiva no pueda alternar entre 1. ${ }^{\mathrm{a}}$ y 3. ${ }^{\mathrm{a}}$, a elección del jugador, y la apreciación de las sincronías se mantenga así más homogénea-y para un solo jugador — la inclusión de más participantes ampliaría las posibilidades del juego y dificultaría aún más la replicación de la ruta jugada-; las desarrolladoras y distribuidoras serán distintas y, por último, también las empresas de localización encargadas de la traducción y el doblaje serán diferentes, en ambos casos, para evitar detectar patrones limitados a una misma empresa, tanto desarrolladora/distribuidora como localizadora, cuyas preferencias en el doblaje pudieran ofrecer resultados asociados a un enfoque particular corporativo, más que al tipo de videojuegos elegidos. De esta forma, los videojuegos que componen finalmente el corpus se vieron reducidos únicamente a tres: Batman: Arkham Knight (BAK), Assassin's Creed Sydicate (ACS) $\mathrm{y}$ Rise of the Tomb Raider (RTR).

La segunda fase la constituye la segmentación del corpus y la alineación entre origen y meta para determinar las relaciones que buscamos entre ambas 
versiones (Laviosa 2012: 68). Como se ha explicado, este corpus multimodal se divide siguiendo, en última instancia, la alternancia continua de situaciones de juego a lo largo de cada uno de los tres juegos analizados. Con algunas salvedades, causadas por el azar presente en todo producto interactivo, la alineación de los segmentos origen y meta, realizada de forma manual recogiendo los datos en columnas contiguas de Excel, resultó exitosa, a pesar de la laboriosa tarea de jugar en ambas versiones, español e inglés, procurando repetir exactamente los mismos pasos en cada uno de los tres juegos. Esta, sin embargo, puede constituir una de las principales limitaciones en un análisis de material interactivo, pues el azar puede causar la no concordancia exacta de segmentos originales y meta en algunos casos. No obstante, en nuestro corpus los porcentajes de no coincidencia son mínimos y, por tanto, poco representativos (las versiones original y meta en BAK coinciden al 96,75\%; en ACS, al $100 \%$ y en RTR, al 99,73\%).

Para estructurar esta segmentación de manera que pudiera accederse al corpus posteriormente de forma cómoda, se grabaron en vídeo las gameplays de cada videojuego en ambas versiones y cada fragmento de vídeo se etiquetó convenientemente, de forma manual, para anotar en el Excel de trabajo dónde se encontraba cada elemento que se iba analizando. Se obtuvieron un total de 300 GB de vídeo, algo más de 76 horas de juego, que recogen 2635 registros con cadenas de texto (1326 en español y 1309 en inglés entre los tres juegos). Este Excel (uno para cada videojuego) constituye la ficha básica de análisis, distribuido de la siguiente manera:

\begin{tabular}{|c|c|c|c|c|c|c|}
\hline $\begin{array}{c}\text { Momento } \\
\text { argumen- } \\
\text { tal }\end{array}$ & $\begin{array}{c}\text { Situación } \\
\text { de juego }\end{array}$ & $\begin{array}{c}\text { Tipo de } \\
\text { ajuste (ES) }\end{array}$ & $\begin{array}{c}\text { Tipo de } \\
\text { ajuste } \\
\text { (EN) }\end{array}$ & $\begin{array}{c}\text { Tipo de } \\
\text { cadena } \\
\text { textual }\end{array}$ & $\begin{array}{c}\text { Comenta- } \\
\text { rios código } \\
\text { de vídeo } \\
\text { (ES) }\end{array}$ & $\begin{array}{c}\text { Comenta- } \\
\text { rios } \\
\text { código } \\
\text { de vídeo } \\
\text { (EN) }\end{array}$ \\
\hline & & & & & & \\
\hline & & & & & \\
\hline
\end{tabular}

Tabla 1. Ficha de análisis

La tercera fase de nuestra metodología, una vez llevada a cabo la extracción de datos, fue realizar las correspondientes generalizaciones de primer nivel sobre las tendencias observadas entre el texto origen y el texto meta. Estas 
apreciaciones las recogemos en el apartado siguiente, referido a los resultados. Como señala Laviosa (2012: 68), será pertinente - y necesario-corroborar estos resultados con futuras ampliaciones del corpus para conseguir mayores niveles de generalización que apunten a tendencias más claras en el uso de las sincronías del doblaje en videojuegos.

Las principales limitaciones que puede presentar un corpus de estas características, además de la ya mencionada, se refieren a su representatividad, a la segmentación y a la transcripción y los métodos de anotación para dar cuenta de su complejidad semiótica (Baños, Bruti y Zanotti 2013; Pavesi 2018).

Aunque la representatividad pueda resultar algo limitada en comparación con otros grandes corpus de cientos de miles de palabras, en este caso debe reconocerse que alcanzar unos criterios de representatividad comunes a todo corpus (multimodal o basado únicamente en texto escrito) no es nada sencillo, dada la ingente cantidad de material y datos que un corpus multimedia genera (Soffritti 2018: 340). Por tanto, siguiendo las recomendaciones de este autor, se han aplicado los filtros ya enumerados al catálogo de videojuegos para reducirlo a un corpus de estudio abarcable que, si bien no da cuenta de una realidad de gran alcance, sí se ha seleccionado en función del fenómeno que se analiza y permite extraer generalizaciones preliminares sobre las que ahondar en futuros trabajos, tal y como se ha hecho anteriormente en otros muchos estudios descriptivos con corpus audiovisuales.

La segmentación, como se ha expuesto, se basa en las situaciones de juego, que representan la unidad de análisis de las sincronías del doblaje y son causa directa y diferenciadora de la presencia de la interacción. Cada situación de juego puede contener diversas cadenas de texto. La alineación presenta ciertas limitaciones inevitables en todo producto sujeto a un considerable grado de aleatoriedad, puesto que, a pesar de reproducir exactamente el mismo camino en las versiones original y traducida, el juego no devuelve siempre respuestas completamente idénticas. Según explica Wolf (2005: 7):

Instead of fixed, linear sequences of text, image, or sound which remain unchanged when examined multiple times, a video game experience can vary widely from one playing to another.

A pesar de ello, en este trabajo nos centramos en las sincronías, un fenómeno que depende del código lingüístico, pero no se limita a este, lo que 
nos permite analizar segmentos, en términos de sincronías, cuyo contenido lingüístico no sea exactamente el mismo.

La transcripción y anotación (véase figura 1) se ha hecho sobre la ficha de análisis (tabla 1) en formato de hoja de cálculo a medida que se ha ido jugando y grabando la gameplay, y también posteriormente. En ella se puede acudir a la herramienta filtros y hacer consultas rápidas, y las anotaciones abarcan todos los canales de comunicación, tanto aspectos visuales relevantes para la configuración de las sincronías como cuestiones acústicas o relativas al tipo de interacción que hayan podido influir en la forma de ajuste empleada. Por ahora, esta es la única opción para gestionar y analizar componentes de audio traducibles en un corpus compuesto por videojuegos, dado que no disponemos de las hojas de cálculo que reciben los traductores ni del guion as rec, según sale del estudio de doblaje. Tampoco podría contarse inicialmente con una transcripción completa del texto, ya que, al ser material interactivo y dinámico, este guion no existe ni siquiera antes de jugar, sino que se va construyendo según la interacción entre el jugador y el juego alternando distintas situaciones. Asimismo, este tipo de análisis con corpus multimodales presenta la limitación de que el texto no puede tratarse mediante ningún tipo de software, al menos por el momento, sino que debe analizarse manualmente, con la lentitud e imprecisión inherentes a este proceso. La figura 1, a continuación, muestra cuatro registros anotados para BAK, a modo de ejemplo.

\begin{tabular}{|c|c|c|c|c|c|c|c|}
\hline 1 & $\begin{array}{c}\text { Momento } \\
\text { argumental }\end{array}$ & $\begin{array}{l}\text { Situación } \\
\text { de inege }\end{array}$ & $\begin{array}{l}\text { Ajuste- } \\
\text { (ES) = }\end{array}$ & $\begin{array}{l}\text { Ajuste: } \\
(\mathrm{EN})=\end{array}$ & Cadena text & Comentarios (ES) & Comentarios (EN) \\
\hline 372 & $\begin{array}{l}\text { Objetivo: Devolver a } \\
\text { los infectados por el } \\
\text { Joker a su celda } 14\end{array}$ & Acción & Libre & Libre & $\begin{array}{l}\text { Enunciados } \\
\text { breves PNJ }\end{array}$ & $\begin{array}{l}\text { Harley Quinn amenaza a Batman } \\
\text { continuamente. Ejemplo de enunciado muy } \\
\text { natural «Por supuesto. ¿Te has enterado, } \\
\text { pedazo de cabrón? ¡Vamos a por til’. } \\
\text { [BAK10_ES 00:36:38] }\end{array}$ & $\begin{array}{l}\text { «You hear that, Bat-freak!? We're } \\
\text { coming to get youls [BAK7_EN } \\
00: 57: 12]\end{array}$ \\
\hline 373 & $\begin{array}{l}\text { Objetivo: Devolver a } \\
\text { los infectados por el } \\
\text { Joker a su celda } 15\end{array}$ & Tarea & Libre & Libre & \begin{tabular}{|l|}
$\begin{array}{l}\text { Instrucciones del } \\
\text { personaje para } \\
\text { el iugador }\end{array}$ \\
\end{tabular} & [BAK10_ES 00:38:40 + 00:39-32] & [BAK7_EN 01:00-02] \\
\hline 374 & $\begin{array}{l}\text { Objetivo: Devolver a } \\
\text { los infectados por el } \\
\text { Joker a su celda } 16\end{array}$ & Cinemática & Sonoro & Sonoro & \begin{tabular}{|l|} 
Enunciado breve \\
PNJ
\end{tabular} & $\begin{array}{l}\text { Quick-time event para contraatacar a la } \\
\text { infectada que se lanza sobre el jugador. } \\
\text { BAK10 ES 00-40:14] }\end{array}$ & [BAK7_EN 01:00-35] \\
\hline 375 & $\begin{array}{l}\text { Objetivo: Devolver a } \\
\text { los infectados por el } \\
\text { Joker a su celda } 17\end{array}$ & Diálogo & Libre & Libre & \begin{tabular}{|l|} 
Diálogo en \\
acción de juego
\end{tabular} & $\begin{array}{l}\text { Robin se queja del ataque. Incoherencia en } \\
\text { la respuesta de Batman «R:...Sé, sincero, } \\
\text { ¿qué pinta tengo? B: Tan lento como tú». } \\
\text { [BAK10_ES 00:40:35] }\end{array}$ & $\begin{array}{l}\text { Ni siquiera mueven los labios. En } \\
\text { inglés: «R. ... Be honest. How's it } \\
\text { look? B: Like you're too slowm. } \\
\text { [BAK7_EN 01:00:55] }\end{array}$ \\
\hline
\end{tabular}

Figura 1. Muestra de la segmentación y anotación de cuatro situaciones de juego en BAK 


\subsection{La triangulación de datos: entrevistas a los agentes de la traducción}

En la búsqueda de tendencias en el uso de las sincronías del doblaje también hemos recurrido al contexto del que emergen los textos multimodales que constituyen el corpus de estudio. La triangulación, según apuntan Taylor y Marchi (2018: 10), es un método valioso para completar el análisis, puesto que tiende a aportar información complementaria que amplía la perspectiva de los datos recogidos, además de ser la base de una investigación sólida que analice el fenómeno en su conjunto (Malamatidou 2018). En este caso, no emplearemos la triangulación basada en la combinación de distintos tipos de corpus, sino, más bien, en la combinación de métodos cuantitativos (el estudio empírico) y cualitativos (entrevistas), en términos de Malamatidou (2018).

Además de la habitual revisión y preparación del marco teórico, de carácter interdisciplinar, dada la polifacética naturaleza de los videojuegos, la consulta de las fuentes textuales se completa con entrevistas semiestructuradas (Kvale y Brinkmann 2009) que dan cuenta de los factores extratextuales referidos a la producción del material analizado.

Dada la estricta confidencialidad bajo la que se trabaja en el ámbito de los videojuegos (Mangiron 2017: 85-86), omitiremos los nombres de las personas entrevistadas, quienes representan la cadena completa de agentes que intervinieron en el proceso de traducción y doblaje para los videojuegos en cuestión. El planteamiento inicial fue entrevistar a todos los agentes desde que se realiza el encargo de traducción hasta que se entrega, es decir, el cliente y la administración (gestores de proyectos) en la preparación del proyecto; traductores y revisores durante la producción; y directores y actores de doblaje, además de los técnicos de sonido, durante la posproducción de la traducción. Sin embargo, los clientes tuvieron que descartarse por la imposibilidad de acceder a ellos. De las 18 figuras restantes, logramos entrevistar a 16, mediante llamada telefónica o videoconferencia, con la posterior transcripción manual de toda la conversación para analizar la información convenientemente.

El diseño de las entrevistas para todos los agentes respondió a tres grandes bloques de preguntas: el perfil de la persona, las indicaciones del encargo 
y el funcionamiento del proceso de traducción y, por último, la fase de doblaje en sala.

La intención de esta herramienta de investigación fue integrar (Malamatidou 2018: 9) la información cualitativa con la extracción empírica para complementar los datos analizados empíricamente, de forma que pudiéramos comprobar si las tendencias observadas se correspondían, en realidad, con la manera en que todos estos profesionales aplicaron las sincronías intencionadamente o si, por el contrario, la aplicación de sincronías es responsabilidad únicamente de algunos de los agentes.

\section{El análisis de las sincronías en nuestro corpus multimodal}

Veamos, pues, cuáles han sido los resultados obtenidos tras delimitar el corpus, los fenómenos analizados y las herramientas de trabajo empleadas. La información cuantitativa resultante del análisis empírico se complementará con el enfoque cualitativo que ofrecen las entrevistas a los agentes del proceso de traducción y doblaje de los tres videojuegos estudiados.

\subsection{Datos cuantitativos extraídos de la ficha de análisis del corpus}

Como se ha indicado, el total de tiempo jugado recogido en las tres tablas de cálculo abarca algo más de 76 horas. De ellas, 26 se corresponden con las versiones original (13 horas) y doblada al español (13 horas) de Batman: Arkham Knight (BAK); 35 horas abarcan las versiones en inglés (17 horas) y en español (18 horas) de Assassin's Creed Sydicate (ACS) y, finalmente, 15 horas se extendió Rise of the Tomb Raider (RTR) en inglés ( 7 horas) y en español (8 horas).

La información extraída del corpus mediante las consultas con filtros en la hoja de cálculo nos devuelve datos cuantitativos sobre las distintas situaciones de juego que componen cada versión de cada videojuego y los tipos de ajuste observados en cada una de ellas. Para sintetizar esta información, se recogen a continuación en la figura 2 y en la tabla 2, numérica, los datos sobre los tipos de ajuste (libre, temporal, temporal exacto, sonoro y labial) detectados en cada situación de juego (tareas, acción, diálogos y cinemáticas) para BAK, ACS y RTR: 


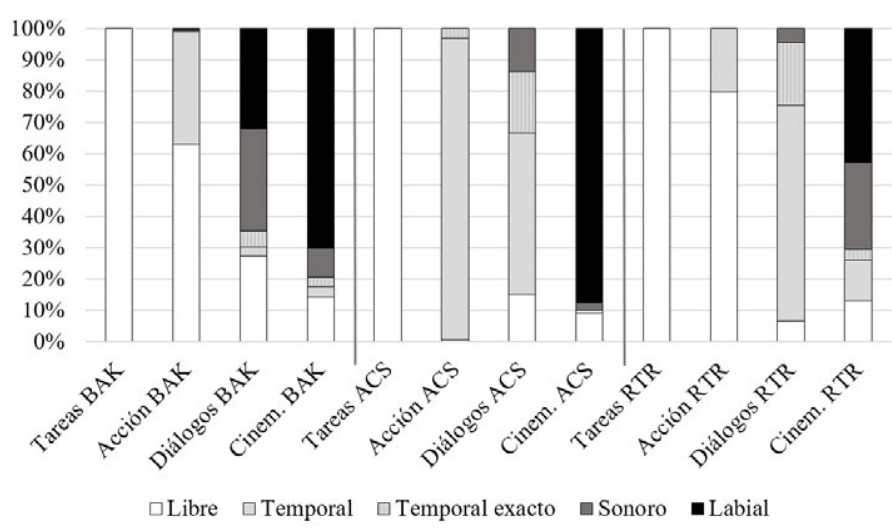

Figura 2. Tipos de ajuste en cada situación de juego de BAK, ACS y RTR

\begin{tabular}{|c|c|c|c|c|c|c|}
\hline & & Libre & Temporal & $\begin{array}{l}\text { Temp. } \\
\text { exacto }\end{array}$ & Sonoro & Labial \\
\hline \multirow{4}{*}{ BAK } & Tarea & $100 \%$ & $0 \%$ & $0 \%$ & $0 \%$ & $0 \%$ \\
\hline & Acción & $63,62 \%$ & $36,32 \%$ & $0 \%$ & $0,53 \%$ & $0,53 \%$ \\
\hline & Diálogos & $27,59 \%$ & $2,87 \%$ & $5,17 \%$ & $32,76 \%$ & $32,18 \%$ \\
\hline & Cinemát. & $14,29 \%$ & $3,06 \%$ & $3,06 \%$ & $9,18 \%$ & $69,39 \%$ \\
\hline \multirow{4}{*}{ ACS } & Tarea & $100 \%$ & $0 \%$ & $0 \%$ & $0 \%$ & $0 \%$ \\
\hline & Acción & $0,62 \%$ & $96,27 \%$ & $3,11 \%$ & $0 \%$ & $0 \%$ \\
\hline & Diálogos & $15,15 \%$ & $51,51 \%$ & $19,7 \%$ & $13,64 \%$ & $0 \%$ \\
\hline & Cinemát. & $8,98 \%$ & $1,2 \%$ & $0 \%$ & $2,39 \%$ & $87,42 \%$ \\
\hline \multirow{4}{*}{ RTR } & Tarea & $100 \%$ & $0 \%$ & $0 \%$ & $0 \%$ & $0 \%$ \\
\hline & Acción & $79,75 \%$ & $20,24 \%$ & $0 \%$ & $0 \%$ & $0 \%$ \\
\hline & Diálogos & $6,67 \%$ & $68,9 \%$ & $20 \%$ & $4,44 \%$ & $0 \%$ \\
\hline & Cinemát. & $13,04 \%$ & $13,04 \%$ & $3,49 \%$ & $27,83 \%$ & $42,61 \%$ \\
\hline
\end{tabular}

Tabla 2. Tipos de ajuste en cada situación de juego de BAK, ACS y RTR

Como se observa en los datos anteriores, parece darse una relación entre determinadas situaciones de juego y tipos de ajuste en estos tres videojuegos de acción-aventura: las tareas, en los tres títulos, presentan siempre un ajuste 
libre, es decir, sin restricciones, lo cual resulta lógico al tratarse siempre de mensajes que se transmiten al jugador de forma diegética mediante voces en off. También en las cinemáticas de los tres juegos parece haber una preferencia clara por el ajuste labial, el más restrictivo y preciso, lo cual enlaza con la naturaleza audiovisual tradicional de este tipo de escenas en las que el videojuego suprime por completo la interactividad y nos traslada a los recursos cinematográficos no interactivos. También hay algunos ejemplos de ajuste libre en las cinemáticas, dado que en todos los juegos se suceden narraciones con voces en off.

La acción de juego es una situación algo más variada: en BAK y RTR hay una clara preferencia por el ajuste libre y bastante presencia del ajuste temporal, mientras que, en el caso de ACS, el uso del ajuste temporal es totalmente predominante. Este dato puede deberse a la propia naturaleza del juego: en BAK y RTR, los personajes emplean continuamente walkies-talkies e intercomunicadores para hablar entre ellos mientras el jugador está actuando, lo cual motiva el uso preferente del ajuste libre, pues se trata de voces en off. En el caso de ACS, al situarse en un medio ${ }^{1}$ histórico, la Londres victoriana de mediados del siglo XIX, la comunicación ha de ser en persona, por lo que el ajuste temporal representa la sincronía más útil en la configuración dinámica e interactiva de la acción con personajes relativamente visibles (aunque no con la precisión que permiten las cinemáticas).

Por último, los diálogos responden a su naturaleza híbrida: en los tres juegos se dan alternativamente con ciertas restricciones de la interacción, cuando se trata de diálogos que el jugador debe escuchar necesariamente, o

1. Debe señalarse que medio no se concibe aquí como el espacio o formato en el que se desarrolla un hecho (televisivo, interactivo, etc.), sino como uno de los distintos criterios que pueden emplearse para clasificar videojuegos (Wolf 2005). Así, entendemos el medio como las convenciones estilísticas, narrativas, temáticas e iconográficas en las que se sitúa la acción (ibid.: 114). Esta concepción podría equipararse a la división tradicional de géneros audiovisuales o narrativos, cuya clasificación no es plenamente extrapolable a los videojuegos, pues estos añaden la interacción entre sus múltiples características. De ahí que los videojuegos puedan clasificarse de forma más precisa según criterios diversos, como el género interactivo (en nuestro caso, acción-aventura, como podría ser también combate, carreras, simulación, estrategia, etc.), el modo de juego (1. ${ }^{\mathrm{a}}$ o 3 . $^{\mathrm{a}}$ persona, o mixto, e individual o multijugador) y el medio (bélico, deportivo, fantástico, terror, etc.), pero no exactamente según el género narrativo tradicional (Wolf 2005; Mejías-Climent 2019: 51-66). 
sin restricción alguna, con interacción plena, cuando se trata de conversaciones de menor relevancia. Por ello, no hay un patrón claro en esta situación de juego cambiante, sino que los cinco tipos de ajuste pueden apreciarse en ellos.

Este análisis cuantitativo representa un punto de partida para continuar explorando en corpus de mayor tamaño. Dadas las tendencias que se apuntan, sería interesante, en primer lugar, ampliar el corpus a más títulos de acción-aventura para comprobar si las tendencias se afianzan. Del mismo modo, podrían analizarse juegos pertenecientes a otros géneros interactivos para comprobar si hay tendencias similares o completamente diferentes.

En segundo lugar, en una selección de videojuegos de acción-aventura podría introducirse la variable del medio en el corpus y comprobar si las tendencias en el uso de las sincronías varían, además, en función del medio en el que se sitúen los videojuegos de acción-aventura (histórico, actual, fantástico o de superhéroes, etc.), pues puede suponerse, a partir de los datos apuntados, cierta relación entre las restricciones del videojuego y la situación contextual y narrativa.

\subsection{Datos cualitativos extraídos de las entrevistas con los agentes}

Por otra parte, como se ha explicado, se quiso comprobar si existe una intención explícita por parte de los traductores o de algún agente de la cadena de traducción y doblaje de estos tres videojuegos a la hora de aplicar distintas sincronías en el texto doblado al español. De este modo, se recurrió a una triangulación metodológica de las herramientas que aportó resultados complementarios (Taylor y Marchi 2018: 7), es decir, que completan la información cuantitativa que se ha obtenido con el análisis empírico.

Las entrevistas semiestructuradas arrojaron luz sobre cada uno de los agentes del proceso de traducción y doblaje (a saber: gestores del proyecto, traductores, revisores, actores de doblaje, directores artísticos y técnicos de sonido) y las tres grandes etapas en las que se lleva a cabo el proceso: preparación y gestión, traducción y revisión lingüística y, por último, doblaje en sala.

En los tres videojuegos, el material que se recibe en la primera etapa se reduce a hojas de cálculo con las cadenas de texto que compondrán los contenidos doblados finalmente en sala, además de cierto material documental 
sobre el videojuego (argumento, personajes y características, mecánicas de juego, especificaciones técnicas, contexto y algunas capturas de pantalla en determinados casos, entre alguna otra información).

Dado el material disponible y tal y como comparten gestores, traductores y revisores, no es posible aplicar sincronías específicas de doblaje en un texto para el cual aún no existen ni siquiera vídeos a los que ajustar la traducción. Por ello, en las etapas de preparación y traducción, las sincronías del doblaje se limitan a restricciones de espacio en función del tipo de cadena, identificadas no exactamente como situaciones de juego, según la terminología investigadora que aquí empleamos, sino, simplemente, como contenido de audio y cinemática (la traducción debe asemejarse lo máximo posible a la extensión del original) y texto en pantalla (las restricciones dependerán exactamente del espacio que se indique en caracteres o palabras, pues se trata de texto que aparecerá en la interfaz del juego).

En la etapa de doblaje en sala es cuando se identifican los cinco tipos de ajuste que analizamos en nuestro corpus, aunque, curiosamente, parece recaer sobre el último agente del proceso, el técnico de sonido, la principal responsabilidad de ajustar al máximo posible las ondas de audio dobladas a las originales, según la situación de la que se trate. Cabe mencionar que tampoco en esta fase se dispone de vídeos finales para los audios que se locutan, aunque sí, en algún caso, pudieron acceder a vídeos de captura de movimiento o motion capture (Kines 2000), o bien, a vídeos degradados para tener una mejor idea de las condiciones en las que se dan ciertas conversaciones, sin poder disponer de los vídeos definitivos.

Los directores artísticos son conscientes de la importancia de ajustar más o menos las ondas de audio a las originales en función del tipo de texto del que se trate, aunque, de nuevo, no emplean exactamente la terminología de las situaciones de juego clasificadas según tareas, acción, diálogos y cinemáticas, sino, más bien, las califican de acción y bloques de onomatopeyas, y escenas cinemáticas, y sí se diferencian los diálogos en RTR. La aplicación de las sincronías parece responder, en buena medida, sobre todo a la experiencia previa en el sector, con proyectos similares, de actores, directores y técnicos de sonido.

La referencia básica en sala de doblaje son las ondas de audio, al no existir vídeo, como sí se tiene al doblar películas o series. El tipo de ajuste 
lo marcan los directores de doblaje según el nivel de restricción del texto: la acción y las onomatopeyas han de asemejarse a las ondas originales con cierto margen (ajuste temporal); en algunos casos, las restricciones son más estrictas (temporal exacto) y, si son cinemáticas reconocidas o diálogos pertenecientes a estas, se procura reproducir con absoluta precisión la onda de audio original, incluyendo pausas, entonación específica o incluso articulación labial (ajuste sonoro y labial).

De nuevo, según su experiencia y el tipo de onda de audio, los técnicos de sonido terminan por ajustar las ondas dobladas al máximo y devuelven a los encargados de la gestión del proyecto una estructura de carpetas y audios idéntica a la que recibieron, pero nunca llegan a ver el resultado final del videojuego doblado y completo, como sí sucede en películas o series, en las que el producto meta sale del estudio de doblaje tal y como se comercializará.

Toda esta información, además de completar los datos de los que ya disponíamos, corrobora la cierta relación que se ha detectado en el corpus entre situaciones de juego y tipos de ajuste, aunque la terminología empleada entre el análisis y estudios anteriores y los profesionales no parece ser exactamente la misma en cuanto a situaciones de juego. Sí se reconocen los cinco tipos de ajuste analizados, aunque, en el caso de los tres videojuegos que componen el corpus, no serán nunca responsabilidad directa del traductor, que está al comienzo de la cadena de traducción y doblaje en videojuegos y solo dispone de texto, nunca de imagen, a diferencia del doblaje cinematográfico.

\section{Conclusiones}

En estas páginas se ha expuesto de forma sintética el análisis de un corpus multimodal (Soffritti 2018) compuesto por videojuegos de acción-aventura que se ha confeccionado expresamente para responder a la pregunta de qué tipos de ajuste se emplean en cada situación de juego. Como se ha explicado, al tratarse de un corpus de estas características, la complejidad en el diseño y la gestión del material es mayor, puesto que se pretende registrar y dar cuenta no únicamente de lo que sucede en el código lingüístico, sino, más bien, en cada segmento (en este caso, situación de juego) analizado en función de su configuración audiovisual completa, transmitida a través de distintos modos semióticos (Kress y Van Leeuwen 2001). En concreto, nos hemos centrado 
en el tipo de ajuste que se emplea en el doblaje de cada una de las cuatro situaciones de juego que representan las unidades de análisis del corpus.

El análisis empírico ha arrojado datos que apuntan a una cierta relación entre las situaciones de juego, que implican diversas formas de interacción con el usuario, y las sincronías del doblaje en videojuegos, concebidas como distintos niveles de restricción. En concreto, parece aplicarse siempre el ajuste libre para las tareas y el ajuste labial en las cinemáticas, con algunas voces en off con ajuste libre. La acción de juego parece depender de varios factores de la configuración del propio juego y del medio en el que se sitúe, pues la preferencia por el ajuste está dividida entre el temporal y el libre. Por último, los diálogos no presentan ninguna preferencia clara, tratándose de una situación híbrida cuya configuración depende por completo de cada juego.

Estos datos se han completado con la información cualitativa extraída de entrevistas semiestructuradas con los principales agentes involucrados en la cadena de traducción y doblaje de los tres videojuegos estudiados. Los resultados apuntan a que las sincronías dependen sobre todo de la última fase de la producción, la locución en sala, y se asocian con distintos momentos del videojuego no identificados como situaciones de juego, sino diferenciados entre acción y onomatopeyas, y algunos diálogos y cinemáticas.

Cabe señalar, como se ha ido mencionando, que este análisis aún ofrece varias limitaciones y representa tan solo un punto de partida para futuras ampliaciones del corpus. Con respecto a las limitaciones, se identifican algunas de las habituales de un corpus situado en el ámbito de la TAV (Baños, Bruti y Zanotti 2013): el registro ha de ser en vídeo apoyado en hojas de cálculo, de forma que se genera una cantidad ingente de datos que hay que administrar convenientemente y que, por el momento, no pueden analizarse mediante ningún tipo de software, más allá de emplear filtros en las hojas de cálculo creadas. En este caso, la segmentación del texto se ha basado en situaciones de juego, cuya alineación entre la versión original y la versión meta puede no ser del todo precisa según el nivel de aleatoriedad que presente el juego como producto interactivo. Esta limitación, no obstante, representará una proporción muy pequeña en corpus de gran tamaño.

Además de ampliar el corpus con videojuegos pertenecientes al mismo género interactivo, la acción-aventura, sería enriquecedor confeccionar 
corpus amplios en otros géneros de videojuegos, de forma que pueda identificarse si en ellos varían o se mantienen las tendencias en el uso de las sincronías del doblaje. De hecho, un estudio de caso preliminar que se está realizando en un videojuego perteneciente al subgénero interactivo de la aventura gráfica (Mejías-Climent 2020) ya apunta a que pueda haber ciertas diferencias, no solo en el uso de las sincronías, sino en la cantidad de situaciones de juego en las que se estructura el corpus, según la naturaleza del videojuego.

Por último, cabe señalar que, para este tipo de estudios de corpus, sería de enorme ayuda contar con las hojas de cálculo originales que se emplearon para las traducciones, pero esta disponibilidad, en especial en el ámbito de los videojuegos, ha de descartarse por completo, dados los estrictos acuerdos de confidencialidad bajo los que las desarrolladoras y empresas de localización trabajan. La cooperación entre industria y academia, una vez más, sería enormemente beneficiosa para asegurar corpus de calidad sobre los que llevar a cabo estudios cuyos resultados fueran prácticos, a su vez, para el mundo profesional. Dada esta carencia, por ahora, el diseño del corpus estudiado empleando situaciones de juego para segmentarlo pretende ofrecer una metodología adecuada a la idiosincrasia multimodal e interactiva del producto, que se irá comprobando con futuras ampliaciones y variaciones en el análisis.

\section{Referencias bibliográficas}

BAKER, Mona. (1996) "Corpus-based translation studies: The challenges that lie ahead." En: Somers, Harold (ed.). Terminology, LSP and translation: Studies in language engineering in honour of Juan C. Sager. Ámsterdam y Filadelfia: John Benjamins, pp. 175-86.

BAÑoS, Rocío, Silvia Bruti y Serenella Zanotti. (2013) "Corpus linguistics and Audiovisual Translation: In search of an integrated approach." Perspectives: Studies in Translatology 21:4, pp. 483-90.

Bernal Merino, Miguel Ángel. (2006) "On the Translation of Video Games." JoSTrans - The Journal of Specialised Translation 6. Versión electrónica: <https://www.jostrans.org/issue06/art_bernal.php>.

Bernal Merino, Miguel Ángel. (2015) Translation and localisation in video games Making entertainment software global. Nueva York: Routledge. 
Bernardini, Silvia y Dorothy Kenny. (2020) "Corpora." En: Baker, Mona y Gabriela Saldanha (eds.). Routledge Encyclopedia of Translation Studies. Londres y Nueva York: Routledge, pp. 110-15.

CADIEUX, Pierre y Bert Esselink. (2004) "GILT: Globalization, Internationalization, Localization, Translation.” Globalization Insider 11:1.5. Versión electrónica: $<$ http://www.il8n.ca/publications/GILT.pdf>.

CAPLE, Helen. (2018) "Analysing the multimodal text." En: Taylor, Charlotte y Anna Marchi (eds.). Corpus Approaches to Discourse. A Critical Review. Londres y Nueva York: Routledge, pp. 85-109.

Chaume, Frederic. (2004a) Cine y traducción. Madrid: Cátedra.

Chaume, Frederic. (2004b) "Synchronization in dubbing: A translational approach." En: Orero, Pilar (ed.). Topics in Audiovisual Translation. Ámsterdam y Filadelfia: John Benjamins, pp. 35-52.

Chaume, Frederic. (2007) "Quality standards in dubbing: a proposal". Tradterm 13, pp. 71-89.

Chaume, Frederic. (2012a) Audiovisual translation: dubbing. Manchester: St. Jerome Publishing.

CHAume, Frederic. (2018a) "An overview of audiovisual translation: Four methodological turns in a mature discipline." Journal of Audiovisual Translation 1:1, pp. 40-63.

Chaume, Frederic. (2018b) "Is audiovisual translation putting the concept of translation up against the ropes?" JosTrans - The Journal of Specialised Translation 30, pp. 84-104. Versión electrónica: <http://jostrans.org/issue30/ art_chaume.php>.

Crosigniani, Simone y Fabio Ravetto. (2001) "Localizing the Buzz! Game Series (Or how to successfully implement transcreation in a multi-million seller video game)." Trans. Revista de Traductología 15 (Special issue on games localization), pp. 29-38. Versión electrónica: <http://www.trans.uma.es/pdf/ Trans_15/29-38.pdf>.

Díaz Cintas, Jorge y Gunilla Anderman. (2009) Audiovisual Translation: Language Transfer on Screen. Houndmills, Hampshire y Nueva York: Palgrave Macmillan.

Dietz, Frank. (2007) "How Difficult Can that be? The Work of Computer and Video Game Localisation." Tradumàtica 5. Versión electrónica: <http://www. fti.uab.es/tradumatica/revista/num5/articles/04/04art.htm>.

EnssLin, Astrid. (2012) The Language of Gaming. Nueva York: Palgrave Macmillan. 
Esselink, Bert. (2000) A Practical guide to software localization. Amsterdam: John Benjamins.

FERNÁNDEZ Costales, Alberto. (2012) "Exploring Translation Strategies in Video Game Localisaton." MonTI 4, pp. 385-408.

FERNÁNDEZ TORNÉ, Anna. (2007) "Anàlisi de la localització de Codename: Kids Next Door - Operation V.I.D.E.O.G.A.M.E.” Tradumàtica 5. Versión electrónica: <http://www.fti.uab.es/tradumatica/revista/num5/articles/08/08art. htm>.

GAMBIER, Yves. (2003) "Screen transadaptation: perception and reception." The Translator 9:2, pp. 171-89.

Hermans, Theo. (2020) "Descriptive translation studies." En: Baker, Mona y Gabriela Saldanha (eds.). The Rotutledge Encyclopedia of Translation Studies. Londres y Nueva York: Routledge, pp. 143-47.

Hurtado Albir, Amparo. (2011) Traducción y Traductología. Introducción a la traductología. 5. ${ }^{a}$ edición. Madrid: Cátedra.

JAKOBSON, Roman. (2000) "On Linguistic Aspects of Translation." En: Venuti, Lawrence (ed.). The Translation Studies Reader. Londres y Nueva York: Routledge, pp. 111-18.

JEwITT, Carey, Jeff Bezemer y Kay O'Halloran. (2016) Introducing Multimodality. Oxon y Nueva York: Routledge.

JiMÉNEZ-CRESPO, Miguel A. (2009) "El uso de corpus textuales en localización."

Tradumàtica 7. Versión electrónica: <http://www.fti.uab.cat/tradumatica/ revista/num7/articles/05/05.pdf>.

JimÉneZ-Crespo, Miguel A. (2013a) "Crowdsourcing, Corpus Use, and the Search for Translation Naturalness: A comparable corpus study of Facebook and non-translated social networking sites." Translation and Interpreting Studies 8:1, pp. 23-49.

JiMÉnEZ-CRESPO, Miguel A. (2013b) Translation and web localization. Oxon: Routledge.

JimÉNEZ-CRESPo, Miguel A. (2015) "Testing Explicitation in Translation: Triangulating corpus and experimental studies." Across Languages and Cultures 16:2, pp. 257-83.

JiMÉnEZ-CRESPO, Miguel A. (2020) "Localization." En: Baker, Mona y Gabriela Saldanha (eds.). The Routledge Encyclopedia of Translation Studies. Londres y Nueva York: Routledge, pp. 299-304. 
KAINDL, Klaus. (2013) "Multimodality and translation.” En: Millán, Carmen y Francesca Bartrina (eds.). The Routledge handbook of translation studies. Londres: Routledge, pp. 257-69.

Kent, Steven L. (2001) The Ultimate History of Video Games. Nueva York: Three Rivers Press.

KINES, Melianthe. (2000) "Planning and Directing Motion Capture for Games." Gamasutra (19 enero). Versión electrónica: <https://www.gamasutra.com/ view/feature/131827/planning_and_directing_motion_.php>.

KRESS, Gunther y Theo Van Leeuwen. (2001) Multimodal discourse. The modes and media of contemporary communication. Londres y Nueva York: Oxford University Press.

KVALE, Steinar y Svend Brinkmann. (2009) Interviewing: learning the craft of qualitative research interviewing. Los Ángeles: Sage.

LAVIOSA, Sara. (2002) Corpus-based translation studies: Theory, findings, applications. Ámsterdam y Atlanta: Rodopi.

LAViosa, Sara. (2012) "Corpora and Translation Studies." En: Hyland, Ken; Chau Meng Huat y Michael Handford (eds.). Corpus Applications in Applied Linguistics. Londres y Nueva York: Continuum International, pp. 67-83.

LÓPEZ REDONDO, Isaac. (2014) ¿Qué es un videojuego? Claves para entender el mayor fenómeno cultural del siglo XXI. Sevilla: Héroes de Papel.

Loureiro Pernas, María. (2007) "Paseo por la localización de un videojuego." Tradumàtica 5. Versión electrónica: <http://www.fti.uab.es/tradumatica/ revista/num5/articles/03/03art.htm>.

Maietti, Massimo. (2004) Semiotica dei videogiochi. Milán: Unicopli.

Malamatidou, (Sofia) 2018. Corpus Triangulation. Combining Data and Methods in Corpus-Based Translation Studies. Londres y Nueva York: Routledge.

MANGIRON, Carme. (2010) "The Importance of not being Earnest: Translating Humour in Video Games." En: Chiaro, Delia (ed.). Translation, Humour and the Media. Londres: Continuum, pp. 89-107.

MAngiron, Carme. (2017) "Research in game localisation." The Journal of Internationalization and Localization 4:2, pp. 74-99.

MATA PASTOR, Manuel. (2005) "Localización y traducción de contenido web." En: Reineke, Detlef (ed.). Traducción y localización: mercado, gestión y tecnologías. Las Palmas: Anroart Ediciones, pp. 187-252.

MaXWell-Chandler, Heather y Stephanie O'Malley Deming. (2012) The Game localization handbook. 2. ${ }^{a}$ edición. Sudbury, Massachussets: Jones. 
Mejías-Climent, Laura. (2017) "Multimodality and dubbing in video games: A research approach." Linguistica Antverpiensia, New Series: Themes in Translation Studies 17, pp. 99-113. Versión electrónica: <https://lans-tts. uantwerpen.be/index.php/LANS-TTS/article/view/463>.

MejÍAs-Climent, Laura. (2018) "El ajuste en videojuegos: el doblaje de Assassin's Creed Syndicate." Trans - Revista de traductología 22, pp. 11-30.

Mejías-Climent, Laura. (2019) La sincronización en el doblaje de videojuegos. Análisis empírico y descriptivo de los videojuegos de acción-aventura. Castellón: Universitat Jaume I. Tesis doctoral inédita.

Mejías-Climent, Laura. (2020) "La evolución de las tecnologías en la confluencia de la interacción y el cine. El doblaje en una aventura gráfica." inTRAlinea. online Translation Journal 22. Versión electrónica: <http://www.intralinea. org/archive/article/2509>.

MÉndeZ GonzÁlez, Ramón y José Ramón Calvo-Ferrer. (2017) Videojuegos y [para]traducción: aproximación a la práctica localizadora. Granada: Comares. MÜller GALHARDI, Rafael. (2014) "Video Games and Fan Translations: A Case Study." En: Mangiron, Carme; Minako O'Hagan y Pilar Orero (eds.). Fun for all: translation and accessibility practices in video games. Berna: Peter Lang, pp. 175-95.

MuÑoz SÁnCHEZ, Pablo. (2017) Localización de videojuegos. Madrid: Síntesis.

Neves, Joselia. (2005) Audiovisual translation: Subtitling for the deaf and hard of hearing. Londres: Roehampton University. Tesis doctoral.

O'Hagan, Minako y Carme Mangiron. (2013) Game localization: translating for the global digital entertainment industry. Ámsterdam: John Benjamins.

VAN OERS, Annelies. (2014) "Translation Strategies and Video Game Translation: A Case Study of Beyond Good and Evil." En: Mangiron, Carme; Minako O'Hagan y Pilar Orero (eds.). Fun for all: translation and accessibility practices in video games. Berna: Peter Lang, pp. 129-48.

Olohan, Maeve. (2004) Introducing corpora in translation studies. Londres: Routledge.

Orero, Pilar (ed.) (2004). Topics in Audiovisual Translation. Ámsterdam y Filadelfia: John Benjamins.

PAVESI, Maria. (2018) "Corpus-based audiovisual translation studies. Ample room for development." En: Perez-González, Luis (ed.). The Routledge Handbook of Audiovisual Translation. Londres y Nueva York: Routledge, pp. 315-333. 
PÉREZ-GonZÁlez, Luis (2020). "Multimodality." En: Baker, Mona y Gabriela Saldanha (eds.). The Routledge Encyclopedia of Translation Studies. Londres y Nueva York: Routledge, pp. 346-351.

PRUYS, Guido Marc. (2009) Die Rhetorik der Filmsynchronisation. Colonia: Eigenverlag.

Pujol Tubau, Miquel. (2015) La representació de personatges a través del doblatge en narratives transmèdia. Estudi descriptiu de pel-lícules i videojocs basats en El senyor dels anells. Barcelona: Universitat de Vic - Universitat Central de Catalunya. Tesis doctoral.

PyM, Anthony. (2014) Exploring Translation Theories. Londres y Nueva York: Routledge.

SOFFritTI, Marcelo. (2018) "Multimodal Corpora and Audiovisual Translation Studies." En: Pérez-González, Luis (ed.). The Routledge Handbook of Audiovisual Translation. Londres y Nueva York: Routledge, pp. 334-349.

TAYLOR, Charlotte y Anna Marchi (eds.) (2018) Corpus Approaches to Discourse. A Critical Review. Londres y Nueva York: Routledge.

TOURY, Gideon (1995) Descriptive translation studies-- and beyond. Filadelfia: John Benjamins.

VÁZQUEZ RodRíGUeZ, Arturo (2018) El error de traducción en la localización de videojuegos. Estudio descriptivo y comparativo entre videojuegos indie y no indie. Valencia: Universitat de València. Tesis doctoral inédita.

Wolf, Mark J. P. (2005) The Medium of the Video Game. 3. ${ }^{a}$ edición. Austin: The University of Texas Press.

Zanettin, Federico. (2014) Translation-Driven Corpora: Corpus Resources for Descriptive and Applied Translation Studies. Oxon y Nueva York: Routledge.

\section{NOTA BIOGRÁFICA / BIONOTE}

LAURA MEJÍAS-CLIMENT es doctora por la Universitat Jaume I (UJI) y licenciada en Traducción e Interpretación por la Universidad Pablo de Olavide. Trabaja en la UJI como docente e investigadora mediante una beca posdoctoral y forma parte del grupo TRAMA. Ha cursado másteres en Traducción Audiovisual, Traducción y Nuevas Tecnologías y enseñanza secundaria e idiomas (MAES). Además, ha impartido clases en la Universidad Pablo de Olavide y en ISTRAD, como profesora del Máster en TAV (Universidad de 
Cádiz) y los másteres en Traducción Especializada (Universidad Internacional Menéndez Pelayo). Participa en el Experto en Traducción y Localización de Videojuegos (ISTRAD) y en el Máster en TAV de la Universidad Europea de Valencia. Trabajó en la University of St. Thomas, en Estados Unidos, mediante una beca Fulbright, y reúne experiencia como traductora profesional y como gestora de proyectos de traducción, especializada en la traducción audiovisual.

Laura Mejías-CLiment holds a PhD in Translation by the Universitat Jaume I (UJI) and a Bachelor's degree in Translation and Interpreting by the Universidad Pablo de Olavide (UPO). She teaches at UJI, where she also works as a postdoctoral researcher and member of the research group TRAMA. She holds three Master's Degrees: in Audiovisual Translation, Translation and New Technologies, and Secondary Education and Languages (MAES). She taught at UPO and ISTRAD, as a lecturer for the Master's Degree in AVT (Universidad de Cádiz) and the master programs in Specialized Translation (UIMP). She participates in the Expert Diploma in Video Game Translation and Localization (ISTRAD) as well as the Master's Degree in AVT at Universidad Europea de Valencia. She also taught in the USA thanks to a Fulbright scholarship, and worked as a translation project manager and a professional translator, specialized in the field of audiovisual translation. 